\title{
Urinary Symptoms after a Routine Pelvic Exam
}

\author{
Jeffrey D. Tiemstra, MD, and Emily Pela, MA
}

Purpose: To determine if urinary symptoms are more common in women during the 4 weeks after a routine pelvic examination.

Methods: This was a 4-week, prospective, observational cohort study in a Family Medicine residency clinic at an urban university. Participants included women ages 18 to 40 years who identified themselves as sexually active and who presented for a routine pelvic examination. Controls were women presenting for other health maintenance with no pelvic examination. Independent variables included age, pelvic examination/no pelvic examination, intercourse frequency, and condom use. Dependent variables included dysuria and urinary frequency. Relative rates of dysuria and frequency were compared using $\chi^{2}$ analysis. Mean rates of dysuria, frequency, intercourse, and condom use were compared using 2-tailed $t$ tests.

Results: Sixty-three subjects and 87 controls completed the study. More subjects had days with dysuria $(17 \%$ vs $7 \% ; P<.01)$, days with frequency $(27 \%$ vs $14 \% ; P<.01)$, days with both dysuria and frequency $(13 \%$ vs $3 \% ; P<.01)$, and days with either dysuria or frequency $(32 \%$ vs $17 \% ; P<.01)$. Subjects had less intercourse than controls $(1.6$ times per week vs 2.2 times per week; $P<.01)$ and used condoms less frequently $(33 \%$ vs $41 \% ; P<.01)$.

Conclusions: Dysuria and urinary frequency are more common among sexually active women during the month after a routine pelvic examination. (J Am Board Fam Med 2011;24:290-295.)

Keywords: Infectious Diseases, Patient Safety, Women's Health

Urinary tract infections (UTIs) are very common among adult women, accounting for more than 6 million physician office visits yearly in the United States. Sexual intercourse has long been recognized as the primary risk factor for female UTI. ${ }^{1}$ Proposed mechanisms for this increased risk include propulsion of bacteria up the urethra into the bladder; microtrauma to the urethra and bladder mucosa, allowing bacterial adhesion; and alteration of normal flora. We postulated that the pelvic examination, including speculum insertion and bimanual examination, may increase the risk of UTIs by similar mechanisms. Although a single pelvic exam-

This article was externally peer reviewed.

Submitted 13 September 2010; revised 15 December 2010; accepted 22 December 2010.

From Family Medicine Center, University of Illinois at Chicago College of Medicine, Chicago.

Funding: This project was supported with a grant from the Joint AAFP/AAFP-F Grant Awards Program G0713.

Conflict of interest: none declared.

Corresponding author: Jeffrey D. Tiemstra, MD, Family Medicine Center (M/C 397), University Village, 722 West Maxwell Street, Suite 235, Chicago, IL 60607 (E-mail: jtiemstr@uic.edu). ination may possibly have only a small effect on an individual's risk of subsequent UTI compared with regular episodes of intercourse, with 20 million Papanicolaou smears annually in the United States and 6 million UTIs, from a public health perspective even a small attributable risk may represent a significant disease burden. A small, retrospective, case-control study suggested such an association. ${ }^{2}$ The present study was designed to prospectively test the theory that urinary symptoms and UTIs are more common among women during the 4 weeks after a pelvic examination.

\section{Methods}

This study was a prospective, controlled cohort study examining the incidence of urinary symptoms for 4 weeks after a pelvic examination screening for Papanicolaou testing. The study was performed at a university-based family medicine residency teaching clinic. The clinic population overall is approximately $40 \%$ college health maintenance organization, $30 \%$ other health maintenance organization, $25 \%$ preferred provider organization/other insurance, and $5 \%$ public aid/uninsured. All participants 
were women ages 18 to 40 years who identified themselves as sexually active.

Subjects were women presenting for a Papanicolaou smear who underwent a screening speculum examination with a bimanual examination. To minimize the effect of inexperienced examiners, only patients of attending physicians, nurse practitioners, and senior residents were eligible for enrollment as subjects. The clinic uses plastic specula, vinyl powder-free examination gloves, and watersoluble lubricating jelly only. Controls were women who presented to the clinic for other health maintenance issues, including office visits for refill of medications (most commonly oral contraceptives), RN visits for immunizations for college/ graduate school, or physicals for school or work not requiring a pelvic examination, and who were otherwise well. Participants were a convenience sample of eligible patients in the clinic during a 24month time frame. On randomly chosen days research staff would screen the schedule for all potentially eligible patients. All potential participants would be approached either before or after their office visit, screened for eligibility, and enrolled. Clinicians were not informed of whether their patients were participating in the study before seeing them.

Once identified as a potential participant, patients were screened for the following exclusion criteria: (1) any current urinary or vaginal symptoms; (2) any chronic urinary or vaginal problems; (3) current treatment with anti-infectives for any nongenitourinary problems; (4) diabetes mellitus; and (5) any chronic medical conditions requiring treatment with steroids or immunosuppressants. Participants were told the study involved looking at risk factors for UTIs but were blinded to the pelvic examination being the factor of interest.

Once enrolled all participants kept a daily checklist for 4 weeks recording intercourse, use of barrier contraception, any dysuria, and any urinary frequency in a yes/no fashion. Study endpoints were completion of the 4-week period or development of a UTI during the 4-week period. Participants were encouraged to seek treatment as they normally would for any symptoms of concern to them. On completion participants returned the checklist in person, at which point it was reviewed for completeness with a research staff member, who also confirmed whether they received any treatment outside the clinic during the study. Participants then received a $\$ 50$ stipend. Charts of participants who completed the study and enrollees who did not complete the study were reviewed to insure that no exclusion criteria were present and that none had been treated for a UTI during the study period that they did not report. The study protocol was approved by the University's Institutional Review Board for research involving human subjects.

\section{Results}

Of 303 patients recruited, 64 subjects and 89 controls completed the study (50\% completion rate). One subject and one control were excluded who had urinary symptoms recorded in their charts on

\section{Figure 1. Participant enrollment.}
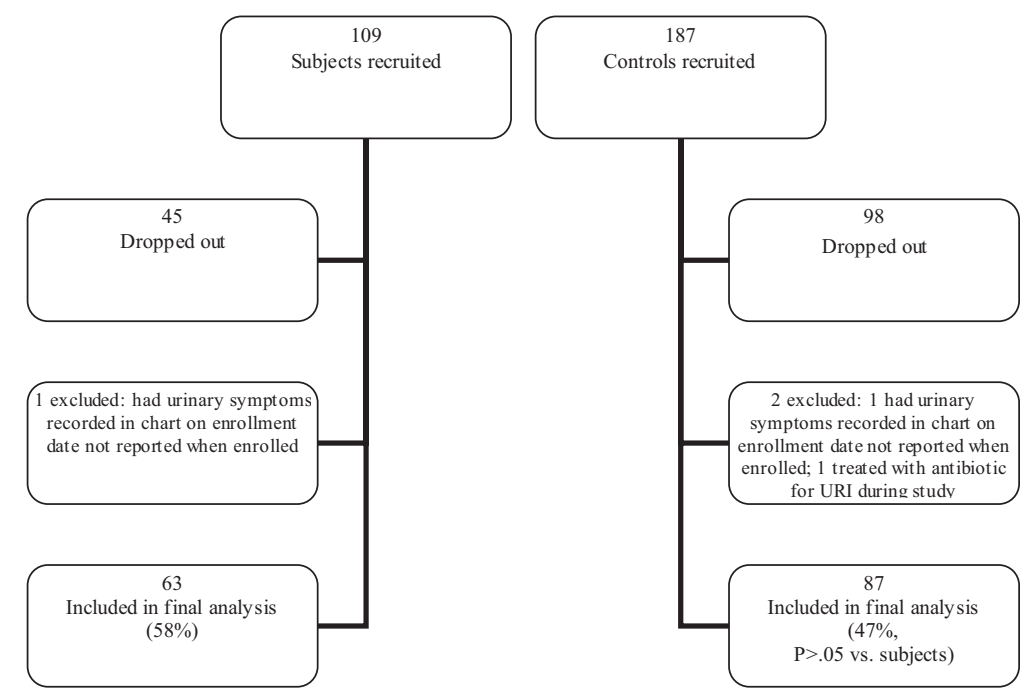
Table 1. Demographic Data Comparing Subjects, Controls, and Dropouts

\begin{tabular}{|c|c|c|c|}
\hline \multicolumn{4}{|c|}{ Number Completing Study } \\
\hline & Subjects & Controls & $\begin{array}{c}\text { Subjects vs. } \\
\text { Controls }\end{array}$ \\
\hline Enrolled & 104 & 187 & \\
\hline Completed Study & $64(59 \%)$ & $89(48 \%)$ & \multirow{2}{*}{$\begin{array}{c}\chi^{2}=3.411 \\
P>.05\end{array}$} \\
\hline Dropped Out & $45(41 \%)$ & $98(52 \%)$ & \\
\hline \multicolumn{4}{|c|}{ Average Age in Years } \\
\hline & Subjects & Controls & $\begin{array}{c}\text { Subjects vs. } \\
\text { Controls }\end{array}$ \\
\hline Completed Study & 26.8 & 26.0 & $P=.32$ \\
\hline Dropped Out & 28.0 & 27.2 & $P=.45$ \\
\hline $\begin{array}{l}\text { Completed vs. } \\
\text { Dropped Out }\end{array}$ & $\mathrm{p}=.28$ & $P=.08$ & \\
\hline
\end{tabular}

the date of enrollment that were not identified at recruitment, and one control was excluded who received antibiotics for a respiratory infection during the study period, for a total 63 subjects and 87 controls included in the final analysis (Figure 1). The average age was 26.8 years for subjects and 26.0 years for controls $(P=.32$; not significant). More controls were recruited than subjects because on any given recruiting day more eligible controls would be seen in the clinic than eligible subjects. Although slightly more subjects than controls dropped out, Table 1 illustrates that the difference in dropout rates was not statistically significant. In addition, there were no significant differences in average age of subjects versus controls who completed the study or who dropped out. Patients of 12 different clinicians were included in the study.

Controls had more intercourse than subjects, with an average of 1.7 days with intercourse per week for subjects versus 2.2 days week for controls $(P=.0004)$. For all participants reporting any barrier contraception use, the charts were reviewed for type of contraception. No participants reported diaphragm use; condoms were the only barrier contraceptive reported by this group. Controls re- ported more condom use, with $33 \%$ of subjects reporting condom use at least once compared with $41 \%$ for controls $\left(\chi^{2}=7.84 ; P<.01\right)$. Controls also reported more consistent condom use: of 21 subjects reporting any condom use, $52 \%$ reported use with every episode of intercourse, whereas of 36 controls reporting any condom use, $61 \%$ reported use every time $\left(\chi^{2}=6.64 ; P<.01\right)$.

Regarding urinary symptoms, significantly more subjects experienced dysuria, urinary frequency, both dysuria and frequency, and either dysuria or frequency than controls (Table 2). One patient in the subject group sought treatment and was diagnosed with and treated for UTI based on symptoms and urinalysis (no culture). No patient in the control group was diagnosed with a UTI during the study period. Subjects and controls with any dysuria averaged 2.4 and 2.0 days of dysuria, respectively $(P=.66$; not significant). Average days with urinary frequency for subjects and controls who reported any frequency were 3.6 and 5.7, respectively ( $P=.32$; not significant).

We hypothesized that the lower rate of intercourse among the subject group may have been because, in part, they were experiencing more urinary symptoms and consequently avoiding intercourse; we tested this hypothesis in 2 ways. First, subjects had a total number of symptomatic days of 1.15 per patient; controls had 0.87 symptomatic days per patient. Correcting the mean frequency of intercourse for subjects of 6.52 per month, using the ratio of symptomatic days yields a corrected intercourse number of 8.57 , very close to the 8.70 observed among the controls. We also compared intercourse frequency in symptomatic and asymptomatic patients, shown in Table 3. Among all participants, the presence of any symptoms was associated with a significantly lower rate of intercourse than the absence of symptoms, although for subjects and controls separately the differences were

Table 2. Total Number of Participants Reporting Dysuria and Urinary Frequency

\begin{tabular}{lcccc}
\hline & Any Dysuria & $\begin{array}{c}\text { Any Urinary } \\
\text { Frequency }\end{array}$ & $\begin{array}{c}\text { Both Dysuria and } \\
\text { Frequency }\end{array}$ & $\begin{array}{c}\text { Any Dysuria, Any Frequency, } \\
\text { or Both }\end{array}$ \\
\hline Subjects* $^{*}(\mathrm{n}=63)$ & $11(17)$ & $17(27)$ & $8(13)$ & $20(32)$ \\
Controls* $\left.^{*}=87\right)$ & $6(7)$ & $12(14)$ & $3(3)$ & $15(17)$ \\
$\chi^{2}$ & 16.02 & 13.28 & 18.34 & 13.35 \\
$P$ & $<.01$ & $<.01$ & $<.01$ & $<.01$ \\
\hline
\end{tabular}

*Data presented as n (\%). 
Table 3. Total Days with Intercourse in Symptomatic Versus Asymptomatic Participants

\begin{tabular}{lccc}
\hline & $\begin{array}{c}\text { Days with } \\
\text { intercourse for } \\
\text { participants } \\
\text { reporting any } \\
\text { symptoms (N) }\end{array}$ & $\begin{array}{c}\text { Days with } \\
\text { intercourse for } \\
\text { participants } \\
\text { reporting no } \\
\text { symptoms (N) }\end{array}$ & $P$ \\
\hline Subjects $(\mathrm{n}=63)$ & $5.7(20)$ & $6.9(43)$ & .141 \\
Controls $(\mathrm{n}=87)$ & $7.9(15)$ & $8.9(72)$ & .385 \\
$P$ & 0.055 & $0.009^{*}$ & \\
All $(\mathrm{n}=150)$ & $6.6(35)$ & $8.1(115)$ & $0.030^{\dagger}$ \\
\hline
\end{tabular}

${ }^{*} P<.01$.

${ }^{\dagger} P<.05$.

not statistically significant. Asymptomatic subjects still had significantly lower rates than asymptomatic controls. Considering other explanations for the lower rate of intercourse among subjects, we also hypothesized that receiving a pelvic examination may have a psychological inhibitory effect on intercourse, independent of any physical symptoms caused by the examination. To test this hypothesis we examined intercourse frequency among subjects who had no physical symptoms after the pelvic examination. Figure 2 shows daily intercourse frequency for all asymptomatic subjects after the pelvic examination and demonstrates no significant change over time.

\section{Discussion}

In this study symptoms of dysuria and urinary frequency were significantly more common among sexually active women in the 4 weeks after a pelvic examination for Papanicolaou screening than in a control group, despite lower rates of intercourse. The lower rate of intercourse among subjects after the pelvic examination may be explained in part by the presence of urinary symptoms. In most women these symptoms clear spontaneously without treatment.

We chose to record only intercourse frequency and barrier contraception use for participants because, among suggested risk factors for UTIs in women, only frequency of intercourse and use of a diaphragm with spermicide have shown strong and consistent associations with UTI risk. ${ }^{3-6}$ Other fac-

Figure 2. Total number of asymptomatic subjects reporting intercourse per day after a pelvic examination.

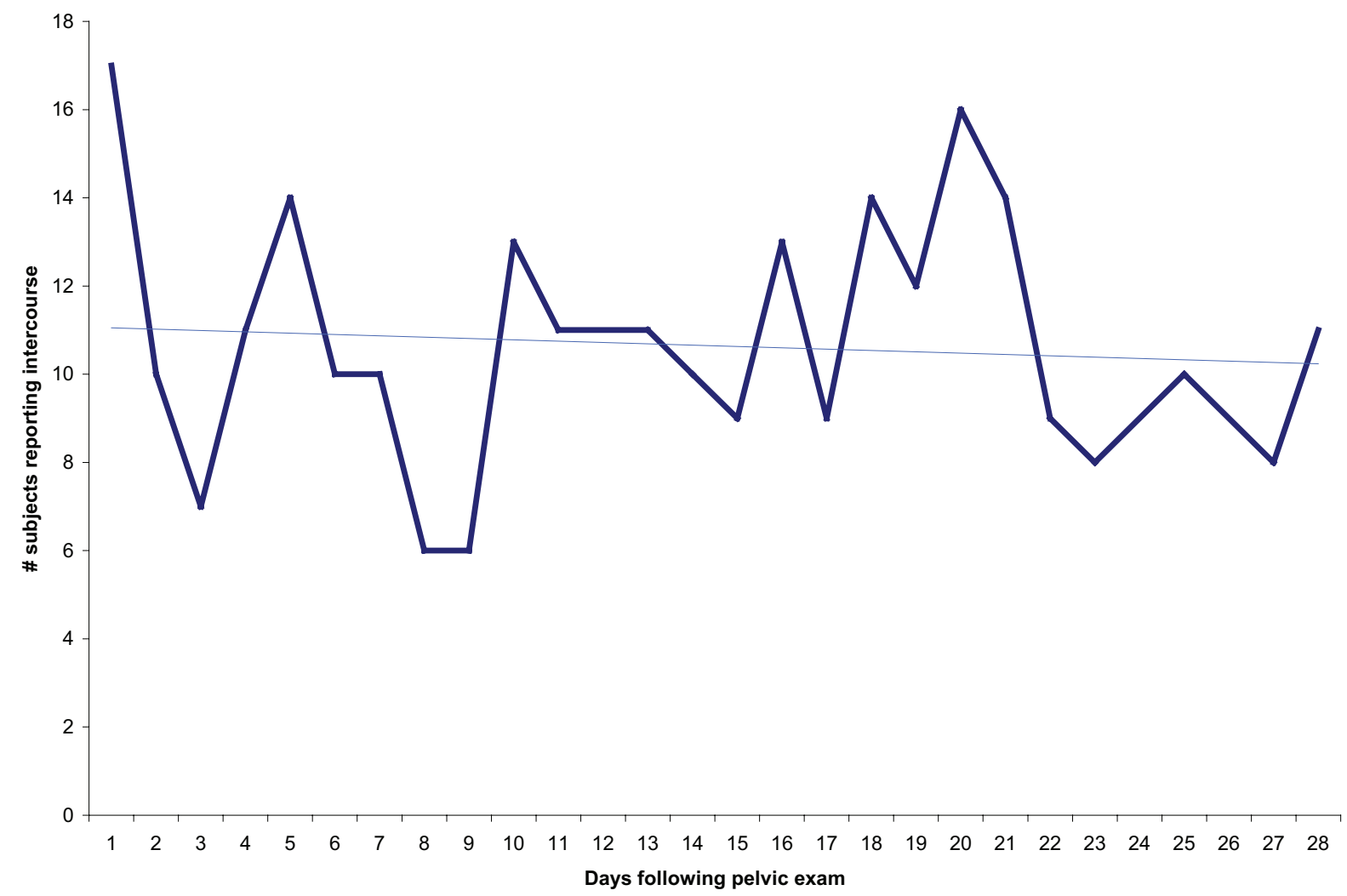


tors such as a new sexual partner, multiple partners, condom use, voiding after intercourse, consumption of acidic juices or carbonated drinks, and use of tampons generally have been found to be weakly or inconsistently associated with UTI risk. Because subjects had a higher rate of symptoms despite a lower rate of intercourse, we believe this offers strong support for the pelvic examination as an independent risk factor for urinary symptoms.

Regarding the timing of symptoms and UTIs after pelvic examination, we chose to follow subjects for 4 weeks to completely capture any potential effect. Previous studies on the timing of UTI risk after intercourse are not completely clear on the risk "window." One study showed an increasing risk of UTI from day 1 to day 5 after intercourse, but did not report on a longer time frame. ${ }^{5}$ Another study reported an increased risk for 2 weeks after intercourse, ${ }^{4}$ and experimental data has shown that significant bacteruria can persist for up to 6 weeks after an initial bacterial inoculum. ${ }^{7}$ Figure 3 shows the timing of symptoms after the pelvic examination for subjects and is consistent with an increasing effect for the first 2 weeks.
The difference in intercourse frequency may be explained in part by the presence of urinary symptoms, but it does raise the question of demographic differences between subjects and controls. Because approximately $40 \%$ of our population is in a college health maintenance organization plan, the control group may have included more college/graduate students, or may have included more patients refilling contraception in anticipation of sexual activity. The college health plan does cover Papanicolaou tests, so many college students were included in the subject group as well. The control group also had a slightly lower (nonsignificant) completion rate. Because this group included patients presenting for simple preventive visits, including $\mathrm{RN}$ visits for immunizations only, this group may have been more mobile and less likely to return to the clinic for their general medical care, or they simply felt less attached to the clinic and less likely to complete the study requirements.

An alternate explanation for the association of symptoms to the pelvic examination would be that subjects entered the study with symptoms they were not reporting. Because clinicians performing a

Figure 3. Total number of subjects reporting symptoms per day after a pelvic examination.

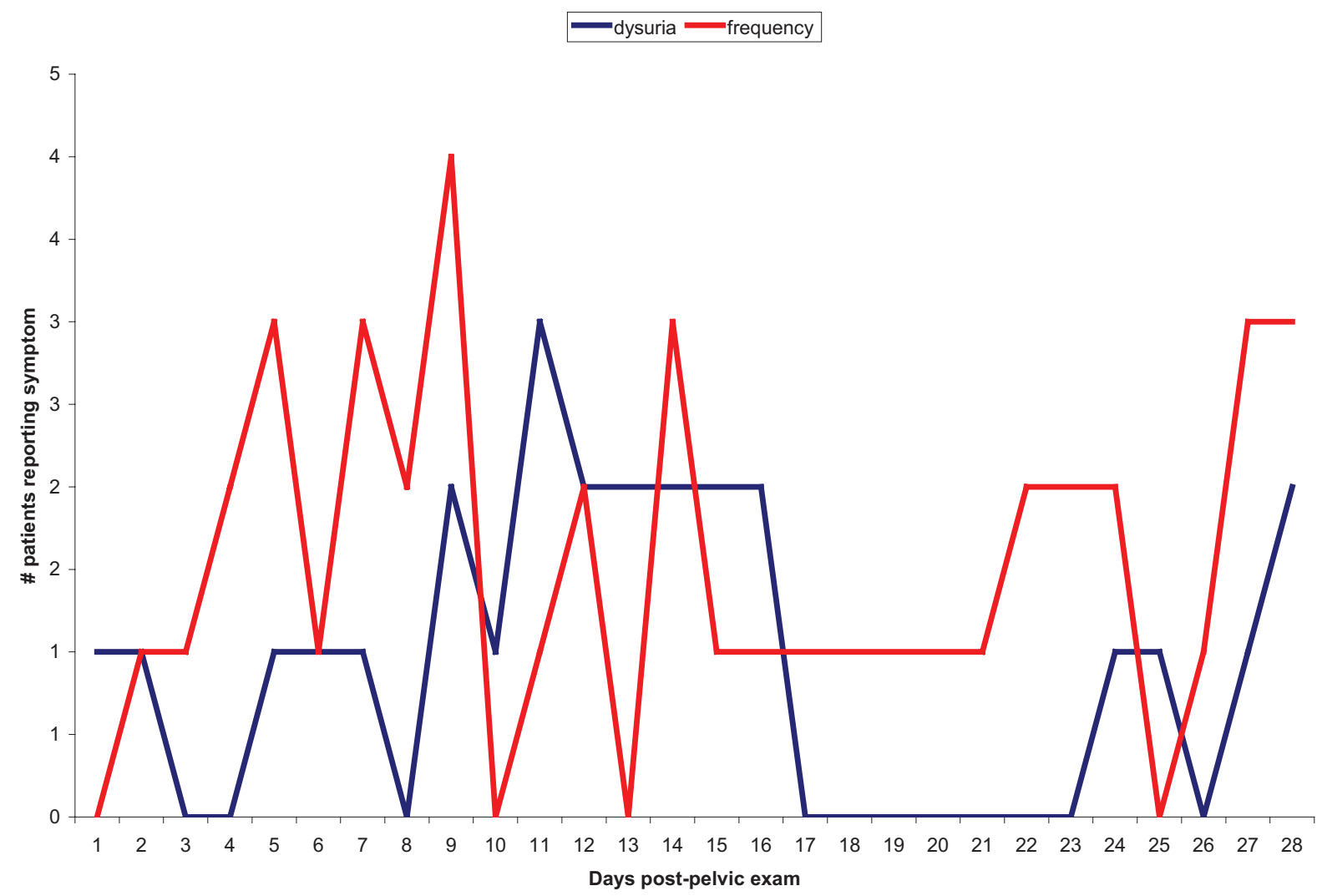


pelvic examination routinely inquire about and record genitourinary symptoms, this explanation seems unlikely. Furthermore, patients who agreed to participate were willing to report symptoms and sexual activity for the study, which makes it unlikely they would be unwilling to report symptoms to their provider.

The finding that urinary symptoms of dysuria and frequency are more common among sexually active women after a routine pelvic examination does not confirm that actual UTI is more common after a pelvic examination. With only one UTI during the 4-week study period in the total participant pool, in retrospect this study was insufficiently powered to measure a difference in confirmed UTI between subjects and controls. That 20 subjects and 15 controls experienced some symptoms but only one sought treatment and was diagnosed with a UTI suggests that many patients will have transient symptoms that clear spontaneously. We do not want to overdiagnose and overtreat patients whose symptoms will clear spontaneously. However, if UTIs are more common after a pelvic examination, then we would want to explore appropriate preventive measures. Simple preventive measures may include following new recommendations for less frequent Papanicolaou screening in women with a history of normal Papanicolaou smears and eliminating the bimanual examination in asymptomatic women since it has little utility as a screening examination.

\section{References}

1. Griebling TL. Urinary tract infections in women. In: Litwin MS, Saigal CS, eds. Urologic diseases in America. Washington, DC: US Government Publishing Office, 2004:153-183.

2. Tiemstra JD, Sinacore JM. The relationship between urinary tract infection and recent pelvic examination in women. Arch Fam Med 1996;5:357-60.

3. Scholes D, Hooton TM, Roberts PL, et al. Risk factors for recurrent urinary tract infection in young women. J Infect Dis 2000;182:1177-82.

4. Foxman B, Geiger AM, Palin K, et al. First-time urinary tract infection and sexual behavior. Epidemiology 1995;6:162-8.

5. Hooton TM, Scholes D, Hughes JP, et al. A prospective study of risk factors for symptomatic urinary tract infection in young women. N Eng J Med 1996;335: 468-74.

6. Foxman B, Chi JW. Health behavior and urinary tract infection in college-aged women. J Clin Epidemiol 1990;43:329-37.

7. Mulvey MA, Schilling JD, Hultgren SJ. Establishment of a persistent Escherichia coli reservoir during the acute phase of a bladder infection. Infect Immun. 2001;69(7):4572-9. 